\title{
THE NON-PARAMETER PENALTY FUNCTION METHOD IN CONSTRAINED OPTIMAL CONTROL PROBLEMS ${ }^{1}$
}

\author{
AN-QING XING \\ University of Regina \\ Department of Mathematics and Statistics \\ Regina, Saskatchewan \\ CANADA S\&S OA2
}

\begin{abstract}
This paper is concerned with the generalization, numerical implementation and testing of the non-parameter penalty function algorithm which was initially developed for solving $n$-dimensional optimization problems. It uses this method to transform a constrained optimal control problem into a sequence of unconstrained optimal control problems. It is shown that the solutions to the original constrained problem. Convergence results are proved both theoretically and numerically.
\end{abstract}

Key Words: Non-parameter penalty function, transform, constrained optimal control, sequence of unconstrained problems.

AMS (MOS) subject classifications: $\quad 49 \mathrm{B36}$

\footnotetext{
${ }^{1}$ Received: September, 1989; Revised: September, 1990.
} 


\section{Introduction}

Penalty function methods were initiated and developed in the area of nonlinear programming ( $\mathrm{cf}$. [1] ). These methods solve a constrained optimization problem via a sequence of unconstrained optimization problems. In recent years, these method have been widely used to solve infinite dimensional optimization problems. Applications of interior and exterior penalty function methods can be found in [3] and [4]. The combination of these two methods forms the so-called mixed penalty function method which has been used by Chen [2] to solve constrained optimal control problems. One difficulty in using these methods is the adjustment of the penalty parameters. In this paper, we apply the non-parameter penalty function method to solve the following constrained optimal control problem:

$$
\min J(u(t))=\min \left[\int_{0}^{T} f_{0}(x(t), u(t), t) d t+L(x(T)]\right.
$$

subject to

$$
\begin{gathered}
\dot{x}(t)=f(x(t), u(t), t), \quad x(0)=x_{I}, \\
g(x(t), u(t), t) \geq 0 \\
h(x(t), u(t), t)=0
\end{gathered}
$$

where $T$ is a fixed positive number and, for each $t \in[0, T]$,

$$
\begin{aligned}
& x(t)=\left(x_{1}(t), \cdots, x_{n}(t)\right) \in R^{n}, u(t)=\left(u_{1}(t), \cdots, u_{r}(t)\right) \in R^{r}, \\
& f(.)=\left(f_{1}(.), \cdots, f_{n}(.)\right) \in R^{n}, g(.)=\left(g_{1}(.), \cdots, g_{m}(.)\right) \in R^{m},
\end{aligned}
$$

and $\quad h()=.\left(h_{1}(),. \cdots, h_{l}().\right) \in R^{l} . \quad f_{k}(k=0, \cdots, n), \quad g_{i}(i=1, \cdots, m) \quad$ and $h_{j}(j=1, \cdots, l)$ are assumed to be continuously differentiable functions on $R^{n+r+1}$. $L($.$) is a continuously differentiable function on R^{n}$. A vector is said to be zero or non-negative if each of its components is.

$u(t)$ is the control of the system and is assumed to be a piece-wise continuous vector-valued function. Its norm can be defined as follows ( cf. [5] ):

$$
\|u(t)\|=\sup _{t \in[0, T]}|u(t)|=\sup _{t \in[0, T]}\left[\left(u_{1}(t)\right)^{2}+\cdots+\left(u_{r}(t)\right)^{2}\right]^{\frac{1}{2}} .
$$

Let

$$
\Omega=\left\{u(t) \mid g_{i}(x(t), u(t), t) \geq 0, i=1, \cdots, m ; h_{j}(x(t), u(t), t)=0, j=1, \cdots, l\right\},
$$


where $x(t)$ is the response corresponding to the control $u(t)$. Then, the constrained optimal control problem is to find a control $u^{*}(t) \in \Omega$ such that

$$
J\left(u^{*}(t)\right)=\min _{u(t) \in \Omega} J(u(t)) .
$$

This is a standard optimal control problem with state variable constraints ( cf. [6] ). The modified maximum principle gives necessary conditions for a control to be optimal ( cf. [6] ). In this paper, let us assume that there exists at least one optimal solution $u^{*}(t)$ and a lower bound $w^{k}$ of the minimum performance measure $J^{*}=J\left(u^{*}(t)\right)$ can be obtained; i. e. a real number $w_{k}$ is known a priori such that

$$
w^{k} \leq J^{*}=J\left(u^{*}(t)\right)
$$

For any control $u=u(t) \in R^{r}$, let

$$
P\left(u, w^{k}\right)=\left(w^{k}-J(u)\right)^{2} G\left(w^{k}-J(u)\right)+J_{1}(u),
$$

where

$$
J_{1}(u)=\sum_{i=1}^{m} \int_{0}^{T}\left(g_{i}(x, u, t)\right)^{2} G\left(g_{i}\right) d t+\sum_{j=1}^{l} \int_{0}^{T}\left(h_{j}(x, u, t)\right)^{2} d t,
$$

and $G(g)=0$ if $g \geq 0$ and 1 if $g<0$. Then, we consider the following unconstrained optimal control problem:

$$
\min P\left(u, w^{k}\right)
$$

subject to (2).

It will be shown how a sequence $\left\{w^{k}\right\}$ of real numbers can be generated automatically by the non-parameter penalty function method. For each $w^{k}$, solve (5) to get a sequence $\left\{u^{k}(t)\right\}$ of unconstrained solutions which converges to a solution to the original constrained optimal control problem (1) - (4).

\section{Theoretical Results}

Since $f_{i}(i=1, \cdots, n)$ are continuously differentiable functions on $R^{n+r+1}$, it can be proved, by the continuous dependence of solutions on parameters, that $J(u)$ is a continuous functional of $u$. Let $u^{k}=u^{k}(t)$ denote the solution of problem (5). Then we have

Theorem 1: Assume that $J_{1}(u)$ satisfies the condition of a "distance function", that is, for any $\bar{u}=\bar{u}(t) \in R^{r}, J_{1}(\bar{u})>0$, and for any $\varepsilon>0$, one can always find a control 
$u=u(t)$ such that

$$
\|u-\bar{u}\| \leq \varepsilon, \quad J_{1}(u)<J_{1}(\bar{u}) .
$$

Then

$$
w^{k} \leq J\left(u^{k}\right) \leq J^{*}
$$

Proof: First, it will be proved that $J\left(u^{k}\right) \leq J^{*}$. Suppose, on the contrary, that $J\left(u^{k}\right)>J^{*}$. Then, since $w^{k} \leq J^{*}$,

$$
P\left(u^{*}, w^{k}\right)=\left(w^{k}-J\left(u^{*}\right)\right)^{2}<\left(w^{k}-J\left(u^{k}\right)\right)^{2} \leq P\left(u^{k}, w^{k}\right) .
$$

This is a contradiction since $u^{k}$ is an optimal solution to problem (5). Hence

$$
J\left(u^{k}\right) \leq J^{*} .
$$

Now, if $w^{k}>J\left(u^{k}\right)$, then $P\left(u^{k}, w^{k}\right)=J_{1}\left(u^{k}\right)$. Since $J(u)$ is a continuous functional, there exists an $\varepsilon>0$ such that $J(u) \leq w^{k}$ for all $u$ satisfying $\left\|u-u^{k}\right\| \leq \varepsilon$. By the assumption of the theorem, an $\bar{u}=\bar{u}(t)$ may be found such that $\left\|\bar{u}-u^{k}\right\| \leq \varepsilon$ and $J_{1}(\bar{u})<J_{1}\left(u^{k}\right)$. Thus,

$$
P\left(\bar{u}, w^{k}\right)=J_{1}(\bar{u})<J_{1}\left(u^{k}\right) .
$$

This contradicts the fact that $u^{k}$ is an optimal solution to problem (5). Therefore,

$$
J\left(u^{k}\right) \geq w^{k}
$$

Theorem 2: Let the assumptions in Theorem 1 hold. If $w^{k}<w^{k+1} \leq J^{*}$, then

$$
J\left(u^{k}\right) \leq J\left(u^{k+1}\right)
$$

where $u^{k+1}=u^{k+1}(t)$ is an optimal solution of (5) with $w^{k}$ replaced by $w^{k+1}$.

Proof: By the definition of $u^{k}$ and $u^{k+1}$,

$$
P\left(u^{k}, w^{k}\right) \leq P\left(u^{k+1}, w^{k}\right), \quad P\left(u^{k+1}, w^{k+1}\right) \leq P\left(u^{k}, w^{k+1}\right) .
$$

Summing the two inequalities gives

$$
\begin{aligned}
& \left(w^{k}-J\left(u^{k}\right)\right)^{2} G\left(w^{k}-J\left(u^{k}\right)\right)+\left(w^{k+1}-J\left(u^{k+1}\right)\right)^{2} G\left(w^{k+1}-J\left(u^{k+1}\right)\right) \\
\leq & \left(w^{k}-J\left(u^{k+1}\right)\right)^{2} G\left(w^{k}-J\left(u^{k+1}\right)\right)+\left(w^{k+1}-J\left(u^{k}\right)\right)^{2} G\left(w^{k+1}-J\left(u^{k}\right)\right) .
\end{aligned}
$$

From Theorem 1 it follows that

$$
J\left(u^{k}\right) \geq w^{k}, \quad J\left(u^{k+1}\right) \geq w^{k+1} .
$$


If $J\left(u^{k}\right)<w^{k+1}$, there is nothing to prove. If $J\left(u^{k}\right) \geq w^{k+1}$, by (6) and (7) it follows that

$$
\left(w^{k}-J\left(u^{k}\right)\right)^{2}+\left(w^{k+1}-J\left(u^{k+1}\right)\right)^{2} \leq\left(w^{k}-J\left(u^{k+1}\right)\right)^{2}+\left(w^{k+1}-J\left(u^{k}\right)\right)^{2} .
$$

That is,

$$
\left(J\left(u^{k}\right)-J\left(u^{k+1}\right)\right)\left(w^{k+1}-w^{k}\right) \leq 0
$$

and, therefore,

$$
J\left(u^{k}\right) \leq J\left(u^{k+1}\right)
$$

Theorem 3: If $w^{k}=J^{*}$, then $u^{k}$ is also a solution to the original problem (1) (4).

Proof: By the assumption,

$$
P\left(u^{k}, w^{k}\right) \leq P\left(u^{*}, w^{k}\right)=0,
$$

where $u^{*}=u^{*}(t)$ is the optimal solution to problem (1) - (4). Since $P\left(u, w^{k}\right) \geq 0$ for any $u=u(t), P\left(u^{k}, w^{k}\right)=0$ and this implies

$$
\left(w^{k}-J\left(u^{k}\right)\right)^{2} G\left(w^{k}-J\left(u^{k}\right)\right)=0, \quad J_{1}\left(u^{k}\right)=0 .
$$

By the definitions of $J_{1}(u)$ and $G(g)$, and by our assumptions on $g_{i}($.$) and h_{j}(),. J_{1}\left(u^{k}\right)=0$ implies that $u^{k}$ satisfies the constraints (2) - (4). Therefore,

$$
J\left(u^{k}\right) \geq J^{*} .
$$

From this and the fact that $\left(w^{k}-J\left(u^{k}\right)\right)^{2} G\left(w^{k}-J\left(u^{k}\right)\right)=0$, it follows that

$$
\left(w^{k}-J\left(u^{k}\right)\right)^{2}=0,
$$

which means $J\left(u^{k}\right)=J^{*}$. Therefore, $u^{k}$ is an optimal solution to problem (1) - (4).

Theorem 4: Let $u^{k}=u^{k}(t)$ be a solution of problem (5). Then

$$
w^{k+1} \stackrel{\Delta}{=} w^{k}+\left[P\left(u^{k}, w^{k}\right)\right]^{\frac{1}{2}} \leq J^{*} .
$$

Furthermore, if $w^{k+1}=w^{k}$ for some $k$, then $u^{k}$ is also a solution to problem (1) - (4).

Proof: By the assumption,

$$
P\left(u^{k}, w^{k}\right) \leq P\left(u^{*}, w^{k}\right)=\left(w^{k}-J^{*}\right)^{2},
$$

and, therefore, 


$$
w^{k+1}=w^{k}+\left[P\left(u^{k}, w^{k}\right)\right]^{\frac{1}{2}} \leq J^{*} .
$$

If $w^{k+1}=w^{k}$, then, by the definition of $w^{k+1}$,

$$
P\left(u^{k}, w^{k}\right)=0 \text {. }
$$

Therefore, $u^{k}$ is an optimal solution to problem (1) - (4) by the proof of Theorem 3.

Theorem 5: If there exists a subsequence of $\left\{u^{k}\right\}$ which converges to some $u^{\infty}=u^{\infty}(t)$, then $u^{\infty}$ is a solution to the original constrained optimal control problem (1) - (4).

Proof: Assume that there exists a subsequence $\left\{u^{i}\right\}$ of $\left\{u^{k}\right\}$ such that

$$
\lim _{i \rightarrow \infty} u^{i}=u^{\infty} \text {. }
$$

Since $\left\{w^{k}\right\}$ is increasing and bounded above $\left(w^{k} \leq J^{*}\right.$ by Theorem 4$)$,

$$
\lim _{k \rightarrow \infty} w^{k}=w^{\infty} \quad \text { or } \quad \lim _{k \rightarrow \infty}\left(w^{k+1}-w^{k}\right)^{2}=0 .
$$

Therefore,

$$
\lim _{k \rightarrow \infty} P\left(u^{k}, w^{k}\right)=\lim _{k \rightarrow \infty}\left(w^{k+1}-w^{k}\right)^{2}=0
$$

In particular,

$$
\lim _{i \rightarrow \infty} P\left(u^{i}, w^{i}\right)=0
$$

Since $P\left(u, w^{k}\right)$ is a continuous functional of $u$ and $w^{k}$, it follows that

$$
P\left(u^{\infty}, w^{\infty}\right)=0 \text {. }
$$

Therefore, $u^{\infty}$ is an optimal solution to problem (1) - (4) by the proof of Theorem 3 .

Theorem 4 implies that if $w^{k}=J^{*}$ we can solve the constrained optimal control problem (1) - (4) by solving one single unconstrained problem (5). In general, it is difficult to know the exact value of $J^{*}$. But, if we can obtain a lower bound $w^{0}$ of $J^{*}$ then we can construct a sequence of unconstrained optimal control problems and solve problem (1) - (4) by solving the sequence. The computing procedure is summarized as follows:

(i) Start from $w^{0}<J^{*}$ and set $k=0$. 
(ii) Solve (5) by some algorithm and get the solution $u^{k}$.

(iii) Calculate $w^{k+1}$ by the formula given in Theorem 4 .

(iv) If $w^{k+1}-w^{k} \leq \delta$, stop and print $u^{k}$. $u^{k}$ is an approximate solution. If $w^{k+1}-w^{k}>\delta$ then replace $w^{k}$ by $w^{k+1}$ and go to (ii). $\delta>0$ is a prescribed tolerance.

\section{A Numerical Example}

Consider the brachistochrone problem with an inequality constraint on the state space:

$$
\min J(u)=\min \left[-x_{1}(T)\right]
$$

subject to

$$
\begin{array}{ll}
\dot{x}_{1}=x_{3} \cos (u), & x_{1}(0)=0, \\
\dot{x}_{2}=x_{3} \sin (u), & x_{2}(0)=0, \\
\dot{x}_{3}=\sin (u), & x_{3}(0)=0.07195,
\end{array}
$$

and the inequality constraint

$$
g\left(x_{1}, x_{2}, x_{3}\right)=0.2+0.4 x_{1}-x_{2} \geq 0,
$$

where $T=1.8$.

This problem was solved in [4] by the exterior penalty function method and the minimum value of $J(u)$ is $J^{*}=-1.0794$. Here we solve this problem by the nonparameter penalty function method by minimizing

$$
P\left(u, w^{k}\right)=\left(w^{k}+x_{1}(T)\right)^{2} G\left(w^{k}+x_{1}(T)\right)+\int_{0}^{T}\left(g\left(x_{1}, x_{2}, x_{3}\right)\right)^{2} G(g) d t
$$

subject to (8) - (10). The Hamiltonian for this problem is

$$
H=g^{2} G(g)+\lambda_{1} x_{3} \cos (u)+\lambda_{2} x_{3} \sin (u)+\lambda_{3} \sin (u),
$$

the adjoint system is

$$
\begin{aligned}
& \dot{\lambda}_{1}=-0.8 g G(g), \quad \lambda_{1}(T)=2\left(w^{k}+x_{1}(T)\right) G\left(w^{k}+x_{1}(T)\right), \\
& \dot{\lambda}_{2}=2 g G(g), \quad \lambda_{2}(T)=0, \\
& \dot{\lambda}_{3}=-\lambda_{1} \cos (u)-\lambda_{2} \sin (u), \quad \lambda_{(T)}=0,
\end{aligned}
$$

and the gradient is 


$$
\frac{\partial H}{\partial u}=-\lambda_{1} x_{3} \sin (u)+\lambda_{2} x_{3} \cos (u)+\lambda_{3} \cos (u)
$$

A Fortran program was written to solve this problem by the gradient method. The numerical integrations are carried out using the fourth-order Runge-Kutta-Gill method and Simpson's composite rule with double precision arithmetic. The integration interval is 0.1 unit.

Numerical results were obtained for $w^{0}=-1.1,-1.2,-1.3$ with convergence index $\delta=0.00001$. The results show that when $w^{0}$ gets closer to $J^{*}$ the convergence gets faster. For $w^{0}=-1.3$ it takes 7 steps in order to get a constrained solution. While, for $w^{0}=-1.1$ only 4 steps are needed. Each step solves an unconstrained optimal control problem and the iteration stops when either the change of the cost function $\left|P^{(i)}-P^{(i+1)}\right| \leq 10^{-6}$ or the norm of the gradient $\|\partial H / \partial u\| \leq 10^{-2}$. At the first step, the initial guess of the control is $u(t)=\pi / 6$. After that, each of the following steps uses the solution obtained at the last step. The trajectories at the steps 1,2 and 7 are shown below. It can be seen that the trajectory at step 7 lies above the constraint line and is almost indistinguishable from the optimal trajectory.

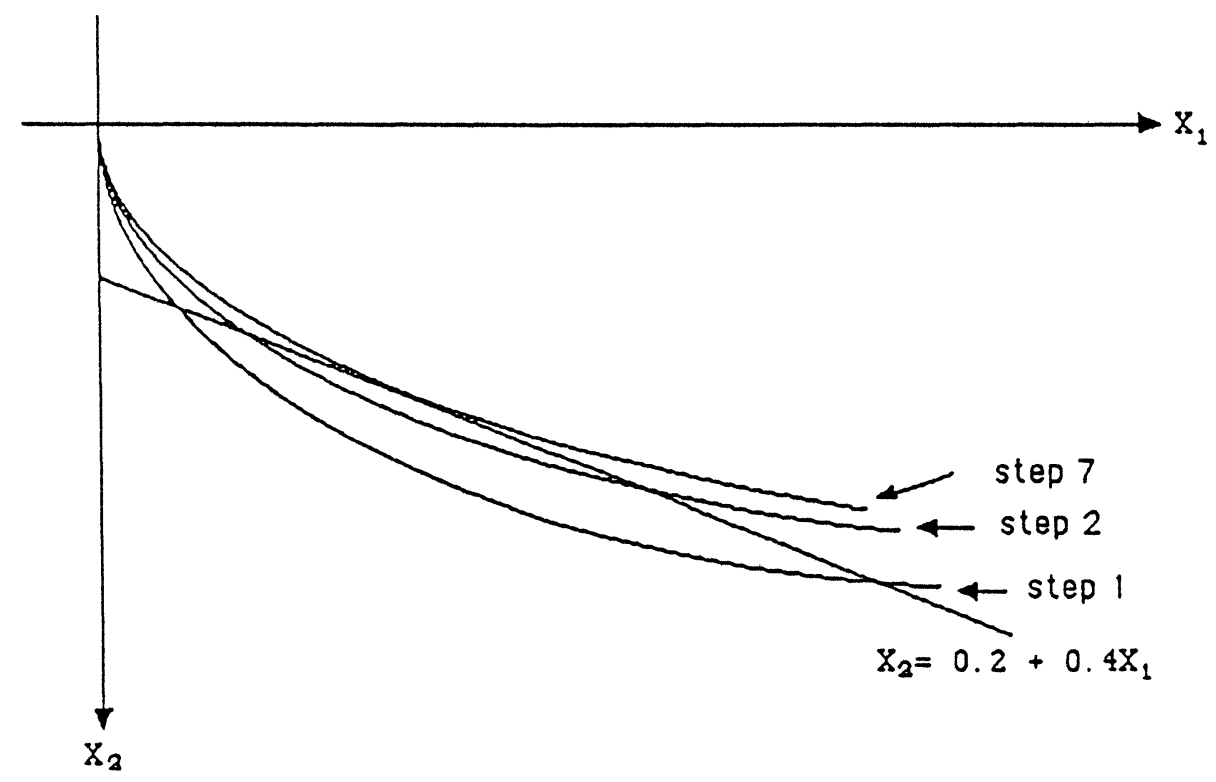

Trajectories at steps 1, 2, and ? 


\section{Summary}

In this paper, we applied the non-parameter penalty function method to solve a constrained optimal control problem via a sequence of unconstrained optimal control problems. Convergence results were obtained. A numerical example was presented to illustrate the findings. The assumption made in Theorem 5 is still an open question and further research will be discussed in other papers.

\section{References}

[1] M. Avriel, Nonlinear programming, Analysis and methods, Prentice-Hall, Inc., Englewood Cliffs, N. J. (1976).

[2] Zuhao Chen, "The mixed penalty function method for solving constrained optimal control problems", Control Theory Appl. 1, 98-109 (1984).

[3] L. S. Lasdon, A. D. Waren and R. K. Rice, "An interior penalty method for inequality constrained optimal control problems", IEEE Tran. Auto. Control, AC12(4), 388-395 (1967).

[4] An-Qing Xing, "Applications of the penalty function method in constrained optimal control problems", J. Applied Mathematics \& Simulation, 2(4), 251-265 (1989).

[5] An-Qing Xing, et al, "Exact penalty function approach to constrained optimal control problems", J. Optimal Control Applications \& Methods, Vol. 10(2), 173180 (1989).

[6] L. S. Pontryagin, The Mathematical Theory of Optimal Processes, Wiley, New York (1962). 


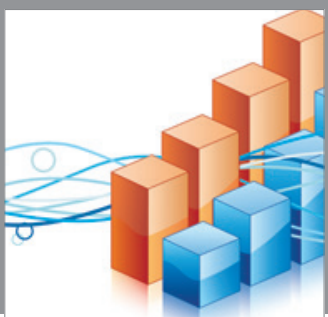

Advances in

Operations Research

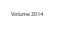

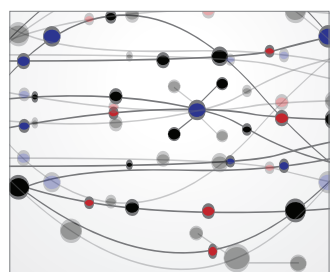

\section{The Scientific} World Journal
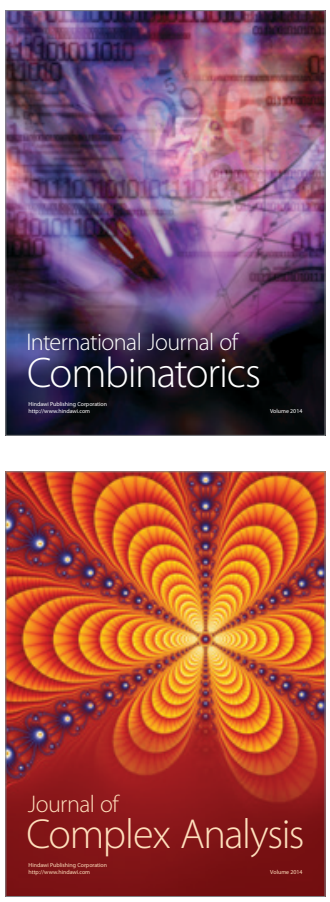

International Journal of

Mathematics and

Mathematical

Sciences
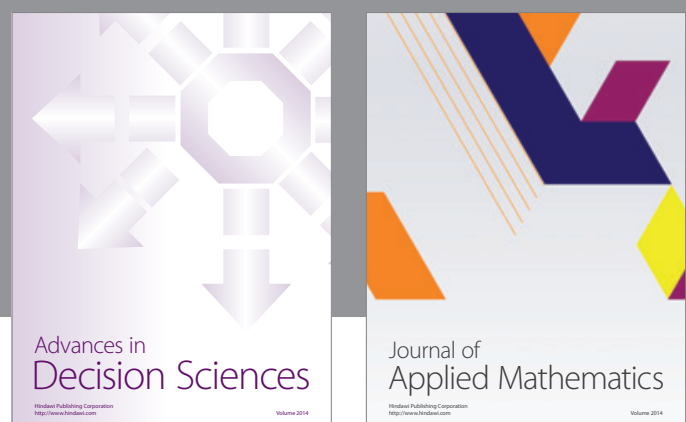

Journal of

Applied Mathematics
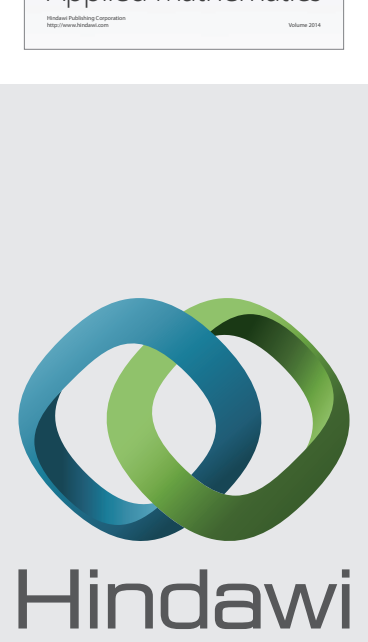

Submit your manuscripts at http://www.hindawi.com
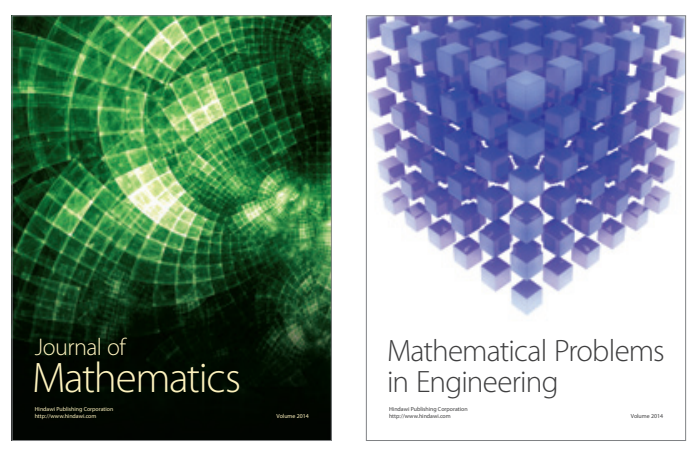

Mathematical Problems in Engineering
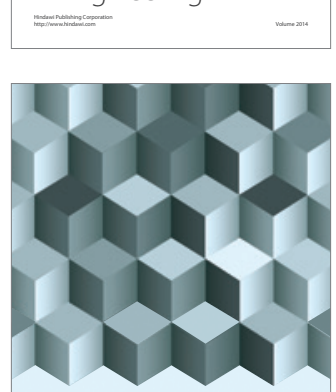

Journal of

Function Spaces
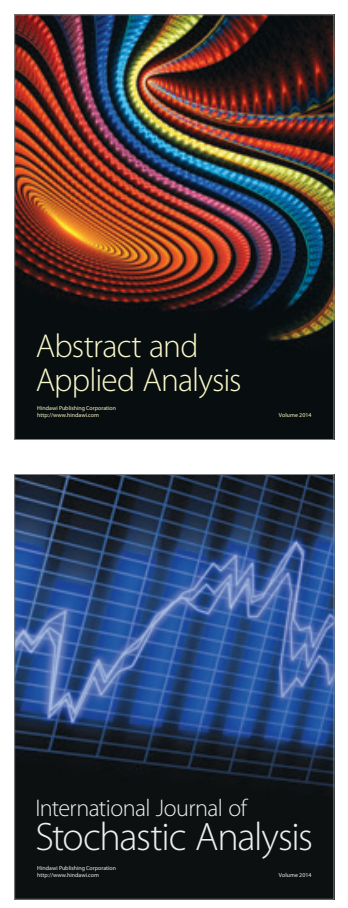

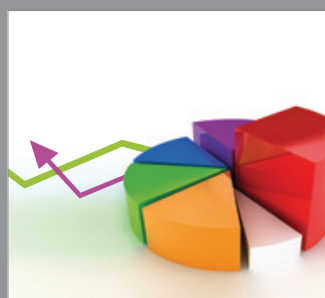

ournal of

Probability and Statistics

Promensencen
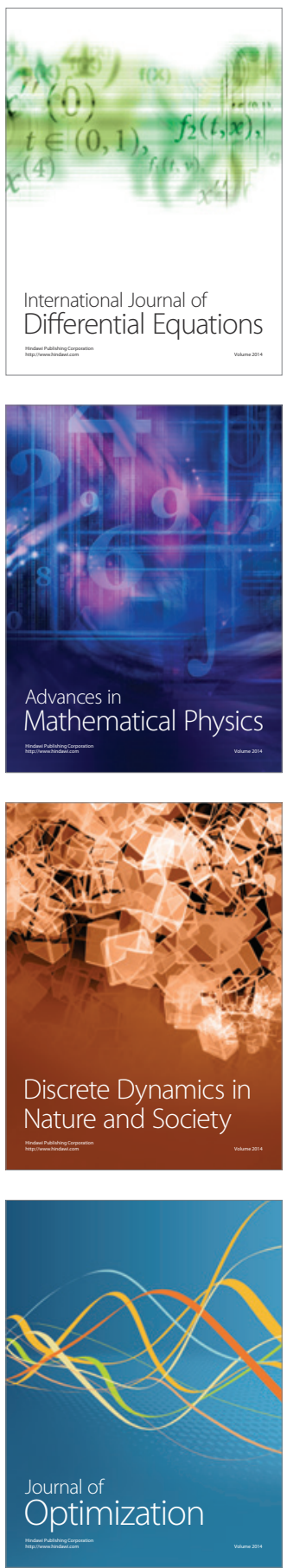